\title{
Realistic MHD numerical simulations of solar convection and oscillations in inclined magnetic field regions
}

\author{
Irina N. Kitiashvili ${ }^{1}$, Alexander G. Kosovichev ${ }^{2}$, Alan A. Wray ${ }^{3}$ and \\ Nagi N. Mansour ${ }^{3}$ \\ ${ }^{1}$ Center for Turbulence Research, Stanford University, Stanford, CA 94305, USA \\ email: irinasun@stanford.edu \\ ${ }^{2}$ Hansen Experimental Physics Laboratory, Stanford University, Stanford, CA 94305, USA \\ email: AKosovichev@solar.stanford.edu \\ ${ }^{3}$ Ames Research Center, Moffett Field, CA 94040, USA \\ email: Alan.A.Wray@nasa.gov and nagi.n.mansour@nasa.gov
}

Keywords. Sun: magnetic fields, oscillations, sunspots; convection, turbulence; methods: numerical

It is known that physical properties of solar turbulent convection and oscillations strongly depend on magnetic field. In particular, recent observations from SOHO/MDI revealed significant changes of the wave properties in inclined magnetic field regions of sunspots, which affect helioseismic inferences. We use realistic 3D radiative MHD numerical simulations to investigate solar convection and oscillations and their relationship in the presence of inclined magnetic field. In the case of highly inclined and strong 1-1.5 kG field the solar convection develops filamentary structure and high-speed flows (Fig. 1a), which provide an explanation to the Evershed effect in sunspot penumbra (Kitiashvili, et al. 2009).
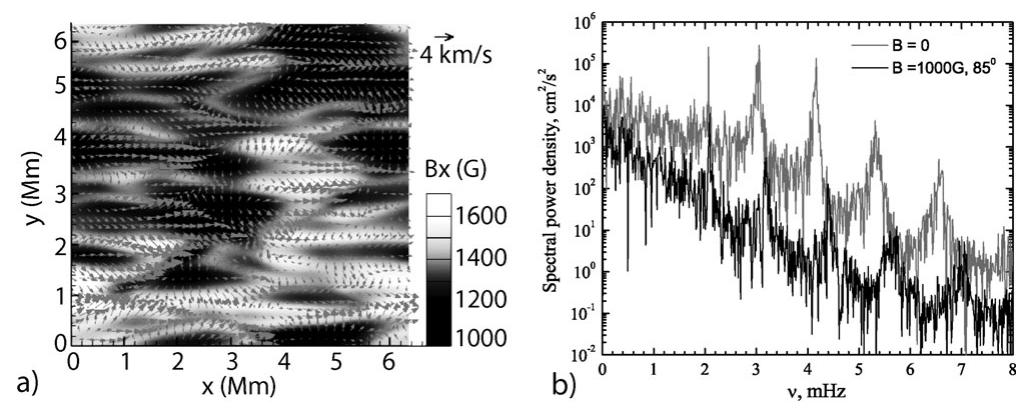

Figure 1.

a) Filamentary structure of the horizontal component of magnetic field, $B x$, developed by magnetoconvection in strong magnetic field $\left(B_{0}=1000 \mathrm{G}\right)$ inclined by $85^{\circ}$ towards the surface. Arrows show the velocity field. b) Oscillation power spectra of the vertical component of velocity averaged over the horizontal photospheric region for the inclined field (black) and non-magnetic region (gray) regions.

The power spectrum of large wavelength oscillations shows that compared to the non-magnetic quiet-Sun regions the amplitude of acoustic oscillations excited by turbulent magnetoconvection in sunspot penumbrae is substantially reduced (at least, by a factor 10), and that the resonant frequencies are shifted towards higher frequencies (Fig.1b). This may explain the observed frequency increase in magnetic regions.

\section{References}

Kitiashvili, I.N., Kosovichev, A.G., Wray, A.A. \& Mansour, N.N. 2009, ApJ 700, L178 